\title{
Theory of Mirror Spectrographs I. Astigmatic Illumination of Plane Gratings and Prisms
}

\author{
Klaus D. Mielenz
}

(July 15, 1964)

\begin{abstract}
By application of an eikonal theory, the image forming properties of plane gratings and prisms are shown to be quite similar. With uncollimated illumination, the astigmatism of both vanishes for an in-plane mounting at the minimum of deviation. Both produce perfect images if illuminated with perfectly collimated (parallel) light. In mirror spectrographs, where this requirement cannot be fulfilled, aberrations are minimized if parallelism of rays is achieved in the cross section normal to the grating grooves, or prism edge.
\end{abstract}

\section{Introduction}

Neither plane gratings nor prisms cause image aberrations when used in perfectly collimated light. Parallel light between collimator and camera, therefore, is standard practice in designing spectrographs. Concave collimating mirrors, however, produce astigmatic pencils of light and, thus, cannot provide the desired perfect collimation. The resulting aberrations of the grating or prism will be examined in this paper. The conditions to minimize these aberrations will be derived.

An eikonal theory will be applied to both plane grating and prism. In principle, this method was already used in H. A. Rowland's [1] ${ }^{1}$ and C. Runge's [2] work on plane and concave gratings. Its modern form is due to P. Zernicke [3] and has subsequently proved to be the most fruitful way to analyze concave gratings $[4,5]$.

The plane grating has been sparingly treated only. Usually, parallel illumination is assumed $[1,4]$. M. V. Murty [6] has studied the plane grating in convergent and divergent light. Astigmatic illumination, however, has apparently never been considered.

An eikonal theory of the prism does not appear to exist at all. Yet, it will be shown here to provide a closed theory of image formation by the latter, also. It also leads to interesting analogies between gratings and prisms.

1 Figures in brackets indicate the literature references at the end of this paper

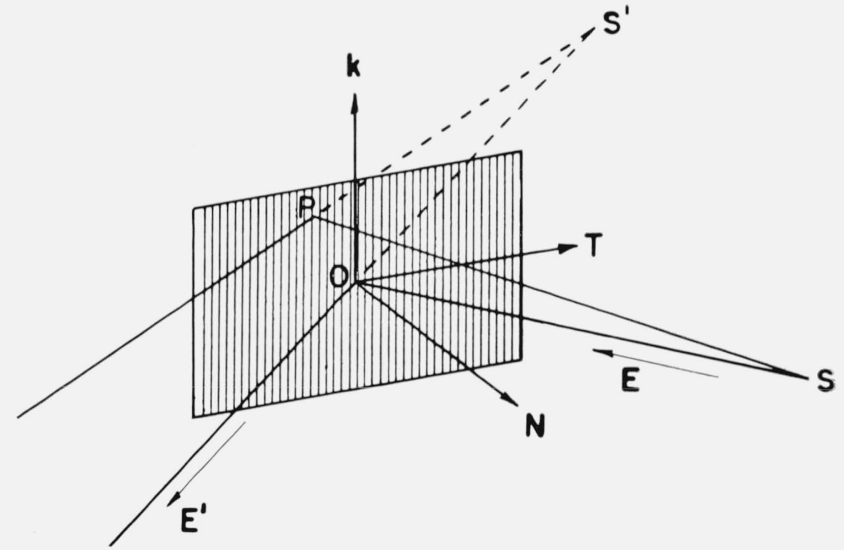

FiguRE 1. Plane reflectance grating.

\section{Plane Grating}

\subsection{Characteristic Function}

Consider a plane reflectance grating illuminated by a point source $S$, as in figure 1 . The grating produces a virtual image, $S^{\prime}$, of the source, and $S^{\prime}$ is a perfect image of $S$ if, for an arbitrary ray, the characteristic function (eikonal)

$$
V=|S P|-\left|P S^{\prime}\right|+p \mu \lambda / d
$$

is independent of the point, $P$, at which the ray intersects the grating (P. Zernicke [3]). Here, $p$ is the coordinate of $P$ in the direction perpendicular to 
the grating grooves, $\mu$ the spectral order, $\lambda$ the wavelength of the diffracted beam, and $d$ the grating constant. As usual in geometrical optics, the virtual path, $\left|P S^{\prime}\right|$, is counted as negative.

Introduce base vectors, $\mathbf{N}, \mathbf{T}$, and $\mathbf{k}$, with the origin at the grating center $O$ so that $\mathbf{N}$ is the grating normal, and $\mathbf{T}$ and $\mathbf{k}$ are the grating tangents perpendicular and parallel to the grooves. Let

$$
\begin{gathered}
\mathbf{S}=-s \mathbf{E}=-s(A, B, C), \\
\mathbf{S}^{\prime}=-s^{\prime} \mathbf{E}^{\prime}=-s^{\prime}\left(A^{\prime}, B^{\prime}, C^{\prime}\right)
\end{gathered}
$$

be the point vectors $\overrightarrow{O S}$ and $\overrightarrow{O S^{\prime}}, \mathbf{E}$ and $\mathbf{E}^{\prime}$ being unity vactors along the principal rays incident and diffracted at $O$, and let

$$
\mathbf{P}=(O, p, q)
$$

be the point vector, $\overrightarrow{O P}$, of $P$. Thus,

$$
\overrightarrow{S P}=-\mathbf{S}+\mathbf{P}, \overrightarrow{P S^{\prime}}=\mathbf{S}^{\prime}-\mathbf{P}
$$

and, for the characteristic function (1),

$$
\begin{aligned}
V & =\sqrt{(\mathbf{S}-\mathbf{P})^{2}}-\sqrt{\left(\mathbf{S}^{\prime}-\mathbf{P}\right)^{2}}+p \mu \lambda / d \\
& =v-v^{\prime}+p \mu \lambda / d .
\end{aligned}
$$

\subsection{Series Expansion}

From (2) and (4),

$$
\begin{aligned}
& v=s\left[1+2(B p+C q) / s+\left(p^{2}+q^{2}\right) / s^{2}\right]^{1 / 2} \\
& =s\left[1+(B p+C q) / s+\frac{1}{2}\left(p^{2}+q^{2}\right) / s^{2}\right. \\
& \quad-\frac{1}{2}(B p+C q)^{2} / s^{2}-\frac{1}{2}\left(p^{2}+q^{2}\right)(B p+C q) / s^{3} \\
& \left.\quad+\frac{1}{2}(B p+C q)^{3} / s^{3}\right],
\end{aligned}
$$

where fourth-order terms are neglected.

Hence, with the corresponding expression for $v^{\prime}$,

$$
\begin{aligned}
V=( & \left.s-s^{\prime}\right)+\left(B-B^{\prime}+\mu \lambda / d\right) p+\left(C-C^{\prime}\right) q \\
& +\frac{1}{2}\left(p^{2}+q^{2}\right)\left(1 / s-1 / s^{\prime}\right) \\
& -\frac{1}{2}\left[(B p+C q)^{2} / s-\left(B^{\prime} p+C^{\prime} q\right)^{2} / s^{\prime}\right] \\
& -\frac{1}{2}\left(p^{2}+q^{2}\right)\left[(B p+C q) / s^{2}-\left(B^{\prime} p+C^{\prime} q\right) / s^{\prime 2}\right] \\
& +\frac{1}{2}\left[(B p+C q)^{3} / s^{2}-\left(B^{\prime} p+C^{\prime} q\right)^{3} / s^{\prime 2}\right] .
\end{aligned}
$$

\subsection{Focus Condition}

The first four terms of (6) vanish, and $V$ is of second-order dependence upon $p$ and $q$ only, if

$$
\begin{gathered}
s=s^{\prime}, \\
B-B^{\prime}+\mu \lambda / d=0, \\
C=C^{\prime} .
\end{gathered}
$$

These equations describe the location of the virtual image in space.

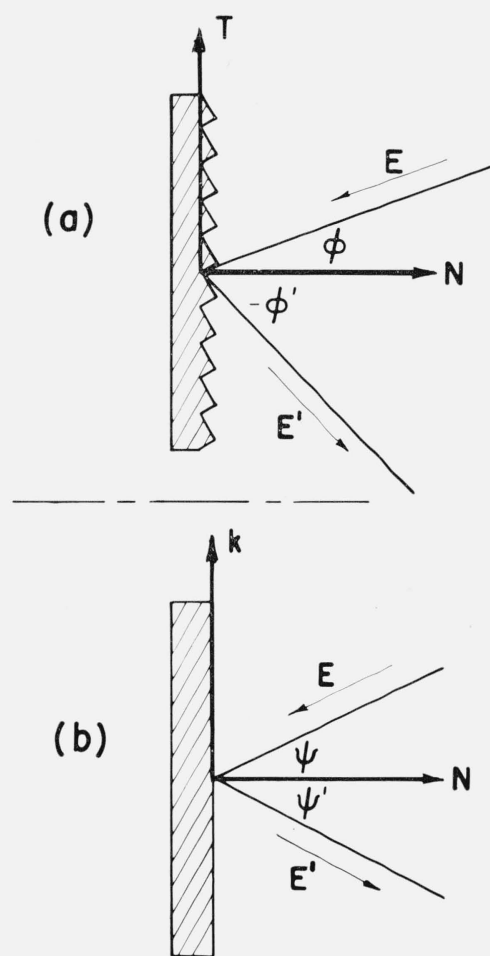

Figure 2. The two principal cross sections of the grating.

For the N, T-plane (fig. 2a), (8) is reduced to the familiar, two-dimensional grating equation,

$$
\sin \phi+\sin \phi^{\prime}=\mu \lambda / d,
$$

since we have, here, $C=C^{\prime}=0$, and

$$
\begin{aligned}
& B=\mathbf{E} \cdot \mathbf{T}=\cos \left(90^{\circ}+\phi\right)=-\sin \phi, \\
& B^{\prime}=\mathbf{E}^{\prime} \cdot \mathbf{T}=\cos \left(90^{\circ}-\phi^{\prime}\right)=\sin \phi^{\prime},
\end{aligned}
$$

where $\phi$ and $\phi^{\prime}$ are the angles which the projections of the principal ray enclose with the grating normal. In accordance with convention, $\phi$ is here always taken as positive, and $\phi^{\prime}$ as positive or negative depending on whether incident and diffracted ray lie on the same or on different sides of the normal.

Equation (9) constitutes the reflection law,

$$
\psi=\psi^{\prime},
$$

for the $\mathbf{N}$, k-plane (fig. 2b), where the grating simply acts as a mirror. For it is, with $B=B^{\prime}=0$,

$$
\begin{gathered}
C=\mathbf{E} \cdot \mathbf{k}=\cos \left(90^{\circ}+\psi\right)=-\sin \psi, \\
C^{\prime}=\mathbf{E}^{\prime} \cdot \mathbf{k}=\cos \left(90^{\circ}+\psi^{\prime}\right)=-\sin \psi^{\prime},
\end{gathered}
$$

where $\psi$ and $\psi^{\prime}$ (both positive) are the angles which the projections of the principal ray enclose with the normal.

$V$ vanishes altogether if, furthermore,

$$
s=\infty .
$$


Used in perfectly collimated light, the grating produces no aberrations.

\subsection{Second-Order Aberrations, Minimum of Deviation}

With (7), (8), and (9), the second-order term of $V$ becomes

$$
-\left[\frac{1}{2}\left(B^{2}-B^{\prime 2}\right) p+\left(B-B^{\prime}\right) C q\right] p / s .
$$

Except for $B=B^{\prime}$ (zero-order reflection from the grating) and for $s=\infty$ (parallel illumination), it vanishes for

$$
B^{\prime}=-B, C=0,
$$

which is the case of the grating used in autocollimation; i.e., of an in-plane mounting at the minimum of deviation. The astigmatism of the grating, therefore, vanishes for this mounting.

\subsection{Higher-Order Aberrations, Astigmatic Illumination}

Throughout the above discussion, illumination of the grating with a spherical or plane wave front was assumed by considering object and image as mathematical points so that the focal distances $s$ and $s^{\prime}$ are the same in all cross sections of the grating. If the grating is however illuminated with an astigmatic pencil of rays; i.e., a cylindrical wave front, different focal distances must be assumed for different cross sections.

Equation (10) can then no longer be satisfied since an astigmatic pencil contains, at best, parallel rays in one cross section only. It must be determined, therefore, how this cross section has to be laid in order that the aberrations of the grating are at least minimized. The two principal cross sections of the grating will be considered separately for this purpose.

N, T-Plane: With $q=0$ and $s=s^{\prime}=s_{H}$, the thirdorder term of (6) is

$$
\frac{1}{2}\left[\left(B^{3}-B^{\prime 3}\right)-\left(B-B^{\prime}\right)\right] p^{3} / s_{H}^{2} .
$$

Except for $B_{1}^{\prime}=B$ and $B_{2,3}^{\prime}=-\frac{1}{2}\left(B \pm \sqrt{4-3 B^{2}}\right),{ }^{2}$ it vanishes for

$$
s_{H}=\infty,
$$

only, when $V$ will be identically zero in the $\mathbf{N}, \mathbf{T}$ plane. Parallel illumination is therefore essential in this cross section.

N, k-Plane: With $p=0, C=C^{\prime}$, and $s=s^{\prime}=s_{V}$, the characteristic function $V$ is identically zero for all choices of $s_{V}$. Parallel illumination, therefore, is not required in this cross section at all.

The aberrations of a grating in astigmatic illumination will thus be minimized if parallelism of rays is only accomplished in the $\mathbf{N}$, T-plane.

\footnotetext{
${ }^{2} B_{1}^{\prime}$ is again the trivial case of zero-order reflection from the grating. $B_{2}^{\prime}$ yields imaginary values of $\phi^{\prime}$. The remaining solution $B$, though real, is not compatible with (11) and therefore of no significance since there is no point in removing third-order aberrations when second-order ones remain uncorrected.
}

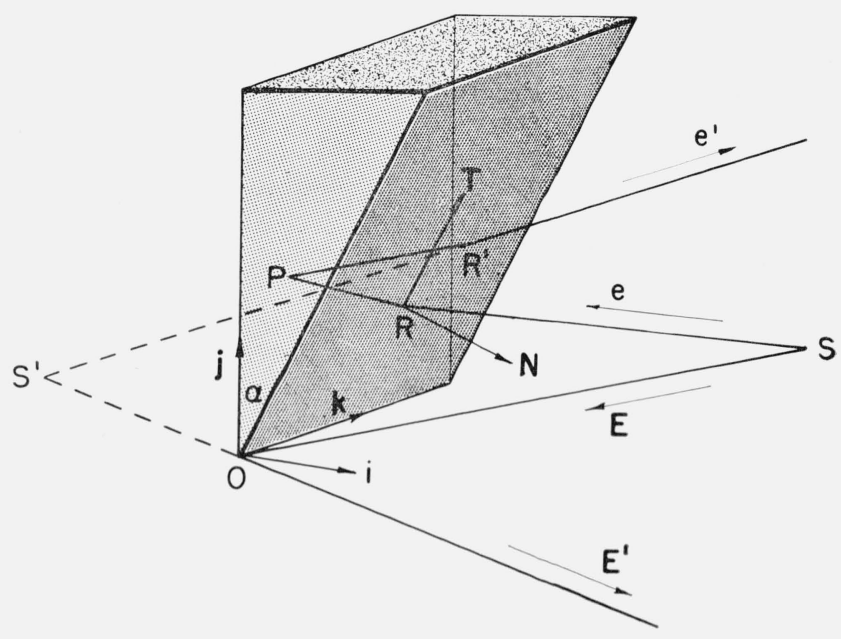

FiguRE 3. Littrow prism.

\section{Prism}

\subsection{Characteristic Function}

Consider a Littrow prism in air illuminated by a point source $S$, as in figure 3 . The prism produces a virtual image, $S^{\prime}$, which is a perfect image if, for an arbitrary ray, the characteristic function

$$
V=|S R|+n\left(|R P|+\left|P R^{\prime}\right|\right)-\left|R^{\prime} S^{\prime}\right|
$$

is independent of the point, $P$, at which the ray intersects the back face of the prism. Here, $R$ and $R^{\prime}$ are the points at which the ray is refracted at the front face, and $n$ is the refractive index of the prism material. The virtual path, $\left|R^{\prime} S^{\prime}\right|$, is counted as negative.

Introduce base vectors, $\mathbf{i}, \mathbf{j}$, and $\mathbf{k}$, with the origin $O$ on the prism edge, so that $\mathbf{i}$ is normal to the back face and $\mathbf{k}$ extends along the edge. Let $\alpha$ be the angle between the two prism faces, so that

$$
\mathbf{N}=(\cos \alpha,-\sin \alpha, 0)
$$

is the normal of the front face and

$$
\mathbf{T}=(\sin \alpha, \cos \alpha, 0)
$$

is the tangent perpendicular to the edge.

Let

$$
\begin{aligned}
& \mathbf{S}=-s \mathbf{E} \\
& \mathbf{R}=\mathbf{S}+|S R| \mathbf{e}, \\
& \mathbf{P}=(O, p, q)=\mathbf{R}+|R P| \mathbf{r}=\mathbf{R}^{\prime}-\left|P R^{\prime}\right| \mathbf{r}^{\prime}, \\
& \mathbf{R}^{\prime}=\mathbf{S}^{\prime}+\left|R^{\prime} S^{\prime}\right| \mathbf{e}^{\prime}, \\
& \mathbf{S}^{\prime}=-s^{\prime} \mathbf{E}^{\prime}
\end{aligned}
$$


be the point vectors $\overrightarrow{O S}, \overrightarrow{O R}, \overrightarrow{O P}, \overrightarrow{O R^{\prime}}$, and $\overrightarrow{O S^{\prime}}$, where $\mathbf{E}$ and $\mathbf{E}^{\prime}$ are the directions of the incident and emerging ray at $O$, e and $\mathbf{r}$ the directions of the incident and refracted ray at $R$, and $\mathbf{r}^{\prime}$ and $\mathbf{e}^{\prime}$ the directions of the incident and emerging ray at $R^{\prime}$.

Thus, as $R$ and $R^{\prime}$ lie on the front face,

$$
\begin{aligned}
& \mathbf{R} \cdot \mathbf{N}=O=-s \mathbf{E} \cdot \mathbf{N}+|S R| \mathbf{e} \cdot \mathbf{N}, \\
& \mathbf{P} \cdot \mathbf{N}=-p \sin \alpha=|R P| \mathbf{r} \cdot \mathbf{N}=-\left|P R^{\prime}\right| \mathbf{r}^{\prime} \cdot \mathbf{N}, \\
& \mathbf{R}^{\prime} \cdot \mathbf{N}=O=-s^{\prime} \mathbf{E}^{\prime} \cdot \mathbf{N}+\left|R^{\prime} S^{\prime}\right| \mathbf{e}^{\prime} \cdot \mathbf{N},
\end{aligned}
$$

and

$$
\begin{gathered}
|S R|=s \mathbf{E} \cdot \mathbf{N} / \mathbf{e} \cdot \mathbf{N}=s U, \\
|R P|=-p \sin \alpha / \mathbf{r} \cdot \mathbf{N}, \\
\left|P R^{\prime}\right|=p \sin \alpha / \mathbf{r}^{\prime} \cdot \mathbf{N}, \\
\left|R^{\prime} S^{\prime}\right|=s^{\prime} \mathbf{E}^{\prime} \cdot \mathbf{N} / \mathbf{e}^{\prime} \cdot \mathbf{N}=s^{\prime} U^{\prime} .
\end{gathered}
$$

The refraction law [7] requires

$$
n(\mathbf{r}-\mathbf{r} \cdot \mathbf{N N})=\mathbf{e}-\mathbf{e} \cdot \mathbf{N N}
$$

or,

$$
n \mathbf{r} \cdot \mathbf{N}=-\sqrt{n^{2}-1+(\mathbf{e} \cdot \mathbf{N})^{2}}=-W<0,
$$

where the negative sign was taken as, in (17b), the length $|R P|$ must be positive.

Likewise,

$$
\begin{gathered}
n\left(\mathbf{r}^{\prime}-\mathbf{r}^{\prime} \cdot \mathbf{N N}\right)=\mathbf{e}^{\prime}-\mathbf{e}^{\prime} \cdot \mathbf{N} \mathbf{N} \\
n \mathbf{r}^{\prime} \cdot \mathbf{N}=+\sqrt{n^{2}-1+\left(\mathbf{e}^{\prime} \cdot \mathbf{N}\right)^{2}}=+W^{\prime}>0
\end{gathered}
$$

and hence, for the characteristic function (13),

$$
\begin{aligned}
V & =\left(s U+n^{2} p \sin \alpha / W\right)-\left(s^{\prime} U^{\prime}-n^{2} p \sin \alpha / W^{\prime}\right) \\
& =v-v^{\prime} .
\end{aligned}
$$

\subsection{Series Expansion}

From (16) through (19),

$$
\begin{aligned}
& \mathbf{S}=-s U \mathbf{e}-|R P| \mathbf{r}+\mathbf{P}, \\
& \mathbf{E}=(U+p \sin \alpha / s W) \mathbf{e} \\
& \quad-\sin \alpha(1+\mathbf{e} \cdot \mathbf{N} / W) \mathbf{N} p / s-(0, p / s, q / s) .
\end{aligned}
$$

Writing,

$$
\begin{gathered}
\mathbf{E} \cdot \mathbf{N}=A, \mathbf{E} \cdot \mathbf{T}=B, \mathbf{E} \cdot \mathbf{k}=C, \\
\mathbf{e} \cdot \mathbf{N}=A / U, p / s=x, q / s=y, \\
(\mathbf{e} \cdot \mathbf{T})^{2}+(\mathbf{e} \cdot \mathbf{k})^{2}=1-(\mathbf{e} \cdot \mathbf{N})^{2}=1-A^{2} / U^{2}, \\
W=\sqrt{n^{2}-1+A^{2} / U^{2}},
\end{gathered}
$$

we obtain, by scalar multiplication of (21) by $\mathbf{T}$ and k, squaring, and adding,

$$
\begin{aligned}
(U+x \sin \alpha / W)^{2}( & \left.-A^{2} / U^{2}\right) \\
& -(B+x \cos \alpha)^{2}-(C+y)^{2}=0,
\end{aligned}
$$

as an implicit representation of $U=U(x, y)$.

From here, the partial derivatives of $U$,

$$
\begin{aligned}
U_{x} & =(\partial U / \partial x)_{0} \\
& =B \cos \alpha-\left(1-A^{2}\right) \sin \alpha / W_{0}, \\
U_{x x} & =\left(\partial^{2} U / \partial x^{2}\right)_{0} \\
& =1-U_{x}^{2}-n^{2} \sin ^{2} \alpha / W_{0}^{2},
\end{aligned}
$$

etc., taken at

$$
x=y=0, U_{0}=1, W_{0}=+\sqrt{n^{2}-1+A^{2}},
$$

can be obtained by repeated implicit differentiation. Then,

$$
U=1+U_{x} x+U_{y} y+\ldots,
$$

whence, by substitution into $(22 \mathrm{~d})$ and further expansion,

$$
1 / W=1 / W_{0}+A^{2}\left(U_{x} x+U_{y} y\right) / W_{0}^{3}+\ldots,
$$

and, from (20),

$$
v=s+\left(W_{0} \sin \alpha+B \cos \alpha\right) s x+C s y+\ldots .
$$

Similarly,

$\left(U^{\prime}-x^{\prime} \sin \alpha / W^{\prime}\right)^{2}\left(1-A^{\prime 2} / U^{\prime 2}\right)$

with

$$
-\left(B^{\prime}+x^{\prime} \cos \alpha\right)^{2}-\left(C^{\prime}+y^{\prime}\right)^{2}=0,
$$

$$
\begin{aligned}
\mathbf{E}^{\prime} \cdot \mathbf{N} & =A^{\prime}, \mathbf{E}^{\prime} \cdot \mathbf{T}=B^{\prime}, \mathbf{E}^{\prime} \cdot \mathbf{k}=C^{\prime}, \\
p / s^{\prime} & =x^{\prime}, q / s^{\prime}=y^{\prime} .
\end{aligned}
$$

Hence,

$$
\begin{aligned}
U_{x^{\prime}}^{\prime} & =B^{\prime} \cos \alpha-\left(1-A^{\prime 2}\right) \sin \alpha / W_{0}^{\prime}, \\
U_{x^{\prime} x^{\prime}}^{\prime} & =1-U_{x^{\prime}}^{\prime 2}-n^{2} \sin ^{2} \alpha / W_{0}^{\prime 2},
\end{aligned}
$$

with

$$
W_{0}^{\prime}=+\sqrt{n^{2}-1+A^{\prime 2}},
$$

and, eventually,

$$
v^{\prime}=s^{\prime}-\left(W_{0}^{\prime} \sin \alpha-B^{\prime} \cos \alpha\right) s^{\prime} x^{\prime}+C^{\prime} s^{\prime} y^{\prime}+\ldots
$$

In the manner thus outlined one obtains by tedious but straightforward calculation, as a third-order approximation for $V$, 
$V=v-v^{\prime}=V_{0}+V_{1} p+V_{2} q+V_{3} p^{2}+V_{4} p q$

$+V_{5} q^{2}+V_{6} p^{3}+V_{7} p^{2} q+V_{8} p q^{2}+V_{9} q^{3}$,

with

$V_{0}=s-s^{\prime}$,

$V_{1}=\left(W_{0}+W_{0}^{\prime}\right) \sin \alpha+\left(B-B^{\prime}\right) \cos \alpha$,

$V_{2}=C-C^{\prime}$,

$V_{3}=\frac{1}{2}\left[\left(1-U_{x}^{2}\right) / s-\left(1-U_{x^{\prime}}^{\prime 2}\right) / s^{\prime}\right]$

$-\frac{1}{2} n^{2} \sin \alpha\left[\left(W_{0} \sin \alpha-2 A^{2} U_{x}\right) / W_{0}^{3} s\right.$

$\left.-\left(W_{0}^{\prime} \sin \alpha+2 A^{\prime 2} U_{x^{\prime}}^{\prime}\right) / W_{0}^{\prime 3} s^{\prime}\right]$,

$V_{4}=-\left(U_{x} C / s-U_{x}^{\prime}, C^{\prime} / s^{\prime}\right)$

$+n^{2} \sin \alpha\left(A^{2} C / W_{0}^{3} s+A^{\prime 2} C^{\prime} / W_{0}^{\prime 3} s^{\prime}\right)$,

$V_{5}=\frac{1}{2}\left[\left(1-C^{2}\right) / s-\left(1-C^{\prime 2}\right) / s^{\prime}\right]$,

$V_{6}=-\frac{1}{2}\left(U_{x} U_{x x} / s^{2}-U_{x^{\prime}}^{\prime} U_{x^{\prime} x^{\prime}}^{\prime} / s^{\prime 2}\right)$

$+\frac{1}{2} n^{2} \sin \alpha\left[A^{2}\left(U_{x x}-3\left(n^{2}-1\right) U_{x}^{2} / W_{0}^{2}\right) / W_{0}^{3} s^{2}\right.$

$\left.+A^{\prime 2}\left(U_{x^{\prime} x^{\prime}}^{\prime}-3\left(n^{2}-1\right) U_{x^{\prime}}^{\prime 2} / W_{0}^{\prime 2}\right) / W_{0}^{\prime 3} s^{\prime 2}\right]$

$V_{7}=-\frac{1}{2}\left[\left(U_{x x}-2 U_{x}^{2}\right) C / s^{2}-\left(U_{x^{\prime} x^{\prime}}^{\prime}-2 U_{x^{\prime}}^{\prime 2}\right) C^{\prime} / s^{\prime 2}\right]$

$-n^{2} \sin \alpha\left[A^{2} U_{x}\left(1+3\left(n^{2}-1\right) / W_{0}^{2}\right) / W_{0}^{3} s^{2}\right.$

$\left.+A^{\prime 2} U_{x^{\prime}}^{\prime}\left(1+3\left(n^{2}-1\right) / W_{0}^{2}\right) / W_{0}^{\prime 3} s^{\prime 2}\right]$,

$V_{8}=-\frac{1}{2}\left[U_{x}\left(1-3 C^{2}\right) / s^{2}-U_{x^{\prime}}^{\prime}\left(1-3 C^{\prime 2}\right) / s^{\prime 2}\right]$

$+\frac{1}{2} n^{2} \sin \alpha\left[A^{2}\left(1-C^{2}-3\left(n^{2}-1\right) C^{2} / W_{0}^{2}\right) / W_{0}^{3} s^{2}\right.$

$\left.+A^{\prime 2}\left(1-C^{\prime 2}-3\left(n^{2}-1\right) C^{\prime 2} / W_{0}^{\prime 2}\right) / W_{0}^{\prime 3} s^{\prime 2}\right]$,

$V_{9}=-\frac{1}{2}\left[\left(1-C^{2}\right) C / s^{2}-\left(1-C^{\prime 2}\right) C^{\prime} / s^{\prime 2}\right]$.

\subsection{Focus Condition}

The first three terms in (25) vanish, and $V$ is of second-order dependence upon $p$ and $q$, only if

$$
\begin{gathered}
s=s^{\prime}, \\
\left(W_{0}+W_{0}^{\prime}\right) \sin \alpha+\left(B-B^{\prime}\right) \cos \alpha=0 \\
C=C^{\prime} .
\end{gathered}
$$

These equations describe the location of the virtual image.

For the $\mathbf{N}$, T-plane (fig. 4a), we have $C=C^{\prime}=0$ and, therefore,

$$
\begin{aligned}
A & =\mathbf{E} \cdot \mathbf{N}=\cos \left(180^{\circ}-\phi\right)=-\cos \phi, \\
A^{\prime} & =\mathbf{E}^{\prime} \cdot \mathbf{N}=\cos \phi^{\prime}, \\
B & =\mathbf{E} \cdot \mathbf{T}=\cos \left(90^{\circ}-\phi\right)=-\sin \phi, \\
B^{\prime} & =\mathbf{E}^{\prime} \cdot \mathbf{T}=\cos \left(90^{\circ}-\phi^{\prime}\right)=\sin \phi^{\prime},
\end{aligned}
$$

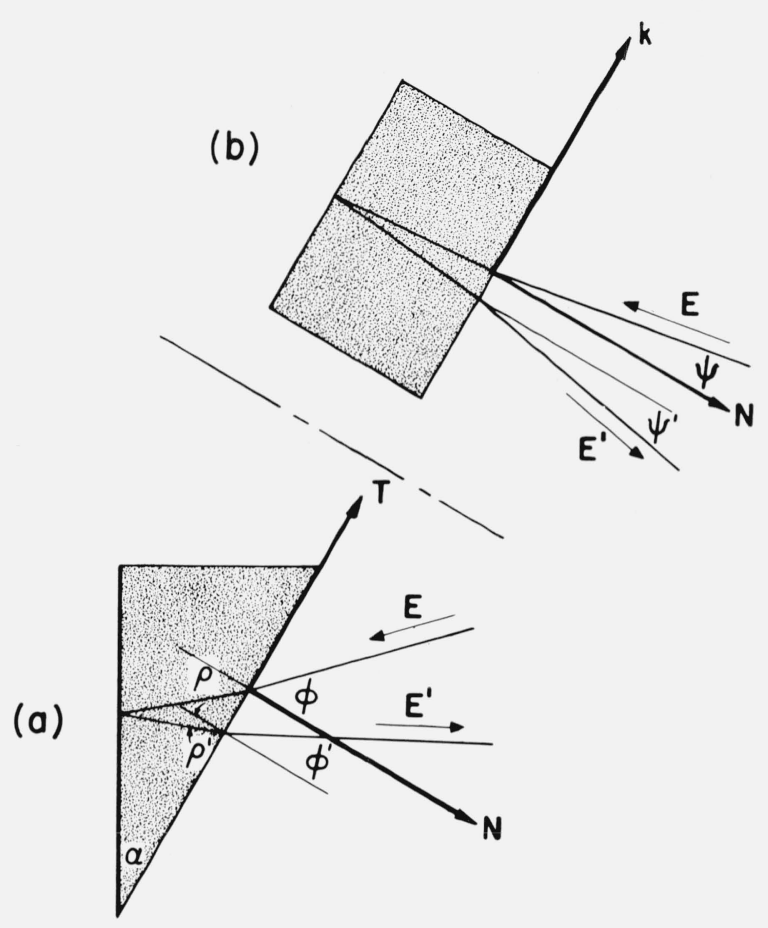

Figure 4. The two principal cross sections of the prism.

(The ray shown is parallel to the ray through $\mathrm{O}$ in fig. 3.)

where $\phi$ and $\phi^{\prime}$ (both positive) are the angles of incidence and emergence in this plane. Thus, from $\left(24 \mathrm{c}, \mathrm{c}^{\prime}\right)$ and $(27)$,

$\sin \alpha\left(\sqrt{n^{2}-\sin ^{2} \phi}+\sqrt{n^{2}-\sin ^{2} \phi}\right)-\cos \alpha\left(\sin \phi+\sin \phi^{\prime}\right)=0$.

With

$$
\sin \phi=n \sin \rho, \sin \phi^{\prime}=n \sin \rho^{\prime},
$$

where $\rho$ and $\rho^{\prime}$ are the angles of refraction pertaining to $\phi$ and $\phi^{\prime}$, this may be written as

$$
n\left[\sin (\alpha-\rho)+\sin \left(\alpha-\rho^{\prime}\right)\right]=0,
$$

which is satisfied if

$$
\rho+\rho^{\prime}=2 \alpha .
$$

Equations (27f and $g$ ) are the familiar relations that describe the passage of a ray through this cross section of the prism.

Equation (28) constitutes the reflection law,

$$
\psi=\psi^{\prime},
$$

for the $N$, $k$-plane (fig. $4 \mathrm{~b}$ ), where the prism simply acts as a mirror. For it is, with $B=B^{\prime}=0$,

$$
\begin{gathered}
C=\mathbf{E} \cdot \mathbf{k}=\cos \left(90^{\circ}+\psi\right)=-\sin \psi, \\
C^{\prime}=\mathbf{E}^{\prime} \cdot \mathbf{k}=\cos \left(90^{\circ}+\psi^{\prime}\right)=-\sin \psi^{\prime},
\end{gathered}
$$


where $\psi$ and $\psi^{\prime}$ (both positive) are the angles of incidence and emergence in this cross section.

$V$ vanishes altogether if

$$
s=\infty .
$$

Illuminated with perfectly collimated light, the prism causes no aberrations.

\subsection{Second-Order Aberrations, Minimum of Deviation}

When the prism is used in autocollimation; i.e., in an in-plane mounting at the minimum of deviation, its astigmatism becomes zero. For one has, in this case,

$$
A^{\prime}=-A, B^{\prime}=-B, C^{\prime}=C=0,
$$

and, with (24a, $\mathrm{a}^{\prime}, \mathrm{c}$, and $\left.\mathrm{c}^{\prime}\right)$,

$$
U_{x^{\prime}}^{\prime}=-U_{x}, W_{0}^{\prime}=W_{0},
$$

so that, from $(25 \mathrm{~d}, \mathrm{e}, \mathrm{f})$ and $(26)$,

$$
V_{3}=V_{4}=V_{5}=0,
$$

for arbitrary values of $s$.

\subsection{Higher-Order Aberrations, Astigmatic Illumination}

With astigmatic illumination, eq (29) can be satisfied in one cross section of the prism, only, and the two principal cross sections of the prism must therefore be considered separately.

N, T-Plane: With $q=0$ and $s=s^{\prime}=s_{H}$, the characteristic function (25) is seen to vanish for

$$
s_{H}=\infty,
$$

only. To eliminate higher-order aberrations in this cross section of the prism, parallel illumination must be used.

N, k-Plane: With $p=0, C=C^{\prime}$, and $s=s^{\prime}=s_{V}$, the characteristic function (25) is identically zero for all choices of $s_{V}$. Parallel illumination is therefore not required in this cross section of the prism.

Hence, the aberrations of the prism will be minimized if parallel illumination is at least provided for the $\mathbf{N}, \mathbf{T}$-plane.

\section{Conclusions}

The above treatment of plane reflectance gratings and Littrow prisms in a like fashion shows that the two dispersive elements act alike in the following respects:

(a) Dispersion takes place in the cross section normal to the grating grooves, or prism edge, only. In the cross section containing the grooves, or edge, the grating and the prism act as plane mirrors.

(b) In an in-plane mounting at the minimum of deviation; i.e., in autocollimation, the astigmatism of the grating, or prism, is zero regardless of illumination.

(c) In perfectly collimated light, the grating and the prism form perfect images.

(d) Illumination with astigmatic light requires parallel rays in the cross section normal to the grating grooves, or prism edge, in order that aberrations are minimized. In the cross section containing the grooves, or edge, deviations from parallelism are permissible.

As reflection from a plane mirror does not at all cause aberrations, the same, or corresponding, results will be obtained by a comparison of transmission gratings and prisms.

While similarities between plane gratings and prisms have been pointed out before [8], it is believed that the above ones are of a less artificial and more intrinsic nature, for they allow the design of grating and prism spectrographs from common principles.

It will be shown in a succeeding paper that the above results have an important influence on the iocal surfaces of mirror spectrographs with either a grating or a prism as the dispersive element.

\section{References}

[1] H. A. Rowland, Phil. Mag. (5) 35, 397 (1893); Astron. and Astrophys. 12, 129 (1893).

[2] See H. Kayser, Handbuch der Spectroscopie, p. 452 (S. Hirzel, Leipzig 1900).

[3] P. Zernicke in Pieter Zeeman, p. 325 (M. Nijhoff, The Hague, 1935).

[4] H. Beutler, J. Opt. Soc. Am. 35, 311 (1945)

[5] T. Namioka, J. Opt. Soc. Am. 49, 446 (1959).

[6] M. V. R. K. Murty, J. Opt. Soc. Am. 5\%, 768 (1962).

[7] M. Herzberger, in Handbook of Physics, p. 6-22 (McGraw-Hill Book Co., New York, N.Y., 1958).

[8] V. von Keussler, Z. Astrophys. 24, 247 (1948).

(Paper 68C4-163) 